\title{
PAPER
}

\section{The natural history and treatment of acquired hemidystonia: report of 33 cases and review of the literature}

\author{
C Chuang, S Fahn, S J Frucht
}

J Neurol Neurosurg Psychiatry 2002;72:59-67

See end of article for authors' affiliations

Correspondence to:

C Chuang, Jacobi Medical Center, 1400 Pelham Parkway S, Room 2E17,

Bronx, NY 10461, USA;

cathychuang@usa.net

Received 12 March 2001

In revised form

3 August 2001

Accepted 24 August 2001
$\mathrm{H}$ emidystonia refers to dystonia which involves the ipsilateral face, arm, and leg. In most cases, a contralateral structural lesion of the basal ganglia or thalamus can be demonstrated by neuroimaging. Common aetiologies include stroke and trauma, and younger patients seem to have a greater propensity to develop hemidystonia after cerebral insults. ${ }^{123}$ There is often a latency between cerebral injury and the onset of dystonia. Hemidystonia may be preceded by ipsilateral hemiparesis which often resolves as hemidystonia develops. Some authors have suggested that symptomatic hemidystonia requires preservation of the corticospinal tract, ${ }^{4}$ and may occur as a result of aberrant reorganisation of the motor system after a static lesion. ${ }^{5}$

Both the natural history and response to treatment of symptomatic hemidystonia have not been extensively studied. As hemidystonia usually follows a single cerebral insult, symptoms might be expected to be relatively static. Hemidystonia has been regarded as poorly responsive to medical therapy, ${ }^{6}$ and surgery has been considered an effective treatment option for such patients. ${ }^{7}$ We evaluated our experience with 33 patients with hemidystonia, and compared our results with 157 cases of hemidystonia previously reported in the literature. Our primary aim was to determine the most effective strategy for treating patients with hemidystonia.

\section{METHODS}

We reviewed the medical records of 33 patients with hemidystonia evaluated at the Movement Disorders Center of Columbia-Presbyterian Medical Center between 1974 and 1999. Age of symptom onset, type of cerebral injury, latency to onset of dystonia, distribution and severity of dystonia, associated symptoms, lesion site (determined by neuroimaging), and response to treatment (medications, botulinum toxin injections, and surgery) were recorded. The distribution and severity of dystonia were determined from physical examination and the Burke-Fahn-Marsden dystonia rating scale. ${ }^{8}$ The effectiveness of medications was determined by the response rate-that is, the percentage of medication trials resulting in any beneficial response as reported by the patient. Follow up of 16 patients $(48 \%)$ by telephone interview included a brief assessment of functional disability.

A Medline search of the world literature revealed 158 cases of hemidystonia reported since 1966. (Case No 15 in our series was previously reported in Burke et al. ${ }^{5}$ ) Data on aetiology, age of symptom onset, latency, results of neuroimaging, and treatment response were collected and compared with our patient series.

\section{RESULTS}

\section{Clinical features}

In our patients $(n=33)$, aetiologies of hemidystonia included stroke ( 10 patients), perinatal injury (nine patients), trauma (eight patients), infection (three patients), congenital lesions (two patients), and tumour (one patient) (table 1). The mean age of dystonia onset was 20 years (range 1-69), and the mean duration of dystonia at last follow up was 15.6 years (range $1-58$ ). In $70 \%$ of patients, symptoms began in childhood or adolescence. Of the 29 patients with a latent period between cerebral insult and onset of dystonia, the mean latency was 4.1 years (range 2 weeks to 17 years), with the shortest latencies occurring in patients after stroke, usually less than 1 year) and the longest latencies occurring after perinatal injury (table 2).

Onset of dystonia was more common in the arm $(48.5 \%)$ than the leg $(21.2 \%)$, and involved the arm and leg simultaneously in $36.4 \%$. The time for dystonia to spread to the 
Table 1 Patient clinical data $(n=33)$

\begin{tabular}{|c|c|c|c|c|c|c|}
\hline $\begin{array}{l}\text { Case/sex/age } \\
\text { of onset }\end{array}$ & History & Latency & $\begin{array}{l}\text { Site of dystonia } \\
\text { onset }\end{array}$ & Spread (time period) & Imaging & Associated signs \\
\hline $1 / F / 52$ & $R$ thalamic bleed post-thalamotomy for torticollis & $4-6$ months & L. hand/foot & None & $R$ thalamic haematoma & L HP, hemisensory loss pain, cramps \\
\hline $2 / F / 6$ & $\begin{array}{l}\text { Normal development, stroke at age 6, angiogram/brain biopsy } \\
\text { normal }\end{array}$ & None & R arm & $R$ leg (23 years later) & L frontal/basal ganglia & R HP, aphasia \\
\hline $3 / M / 70$ & Stroke at age 70 & 3 months & L hand/foot & None & $R$ thalamic infarct & LHP, pain \\
\hline $4 / F / 66$ & Stroke, L HP for 1 day & 8-9 months & Larm & L foot/jaw/pharynx (1 y) & R MCA infarct & L HP, pain, contracture \\
\hline $5 / F / 2$ & Stroke at age $2, \mathrm{~L} \mathrm{HP}$ improved in $5-6 \mathrm{~h}$ & Several weeks & L hand & L leg (soon after) & R basal ganglia (putamen/caudate) & LHP \\
\hline $6 / M / 61$ & L basal ganglia bleed & None & $R$ hand & $\mathrm{R}$ arm/foot & L basal ganglia haemorrhage & RHP \\
\hline $7 / M / 56$ & 2nd stroke with L HP & 5 months & L arm & L foot (N/A) & $R$ parieto-occipital infarct & L HP/hemisensory loss \\
\hline $8 / F / 27$ & $\begin{array}{l}\text { TIA with L HP (age 24) normall LP, angiogram, imaging. } 3 \text { years later, } \\
\text { had MVA }\end{array}$ & 3 y & L foot & L hand (soon after) & $\operatorname{scan} N / A$ & \\
\hline 9/M/59 & L thalamic/basal ganglia haemorrhage & 6 months & R arm/foot & None & L thalamic/basal ganglia haemorrhage & R HP, aphasia, dysaesthesia \\
\hline $10 / \mathrm{M} / 6$ & $\begin{array}{l}\text { L HP at age 5, Dx R midbrain tumour extending to thalamus, } \\
\text { underwent XRT/shunt }\end{array}$ & 9 months & R leg & R arm/torso (6 months) & L putamen infarct & Pain \\
\hline $11 / F / 39$ & MVA with head trauma, age 38 & $1 \mathrm{y}$ & L hand & $\mathrm{L}$ leg ( 3 years later $)$ & $\begin{array}{l}R \text { putamen/parietal insular, ant temp. } \\
\text { encephalomalacia }\end{array}$ & $\begin{array}{l}\text { L HP, hemisensory loss, cognitive } \\
\text { dysfunction, depression }\end{array}$ \\
\hline $12 / M / 13$ & Fishing pole impaled eye, coma for 2 weeks & 4 months & R arm/foot & None & L haematoma & R HP, aphasia \\
\hline 13/F/5 & Head trauma without LOC, R parietal laceration & 1 month & R arm & $R$ foot (6 months later) & N/A & R HP \\
\hline $14 / F / 8$ & Fell off bicycle, head trauma without LOC & 3 months & R arm/neck & $R$ foot & L basal ganglia infarct & None \\
\hline $15 / F / 9$ & MVA with depressed skull fracture, age 2 ; coma for 10 days & $6 y$ & R arm/leg & None & N/A & R HP \\
\hline $16 / \mathrm{M} / 11$ & $\begin{array}{l}\text { Fell off horse, head trauma, } 3 \text { days later R HP/sensory loss, no LOC, } \\
\text { recovery in } 2 \text { weeks }\end{array}$ & 2 weeks & R arm/leg & None & N/A & R HP, R HH, hemisensory loss \\
\hline $17 / F / 23$ & Fell down stairs, hit right side of head, no LOC & 8 months-1 y & L hand & L foot/arm/leg (several years) & Thalamotomy lesion & Increased tone \\
\hline $18 / F / 24$ & Fell off horse with mild head injury & $10 y$ & R finger & $\mathrm{R}$ arm $/ \mathrm{leg} /$ voice (1 y later) & L midbrain & Dysarthria \\
\hline 19/F/9 & $\begin{array}{l}\text { Born at } 28 \text { weeks, C-S Apgars } 0 \text { and } 1 \text {, intubated, NICU 21/2 months, } \\
\text { global delay }\end{array}$ & $9 y$ & L foot & L wrist/arm, R arm (9y) & $\begin{array}{l}\text { Dandy-Walker (cerebellar/pons hypoplasia) global } \\
\text { cerebral atrophy }\end{array}$ & Cognitive self mutilation behaviour \\
\hline $20 / M / 1$ & Forceps delivery, Apgars normal, pneumonia in pregnancy & $1 y$ & $\mathrm{~L}$ leg & L arm (soon after) & N/A & Shoulder pain \\
\hline $21 / M / 8$ & $\begin{array}{l}\text { PROM at } 5 \text { months, forceps delivery at } 6 \text { months, NICU } \\
21 / 2 \text { months, seizures }\end{array}$ & $8 y$ & L hand & L leg & Deep WM of R temporal lobe superior to putamen & None \\
\hline $22 / M / 13$ & Neonatal asphyxia, intubated, seizures, incoordination as a child & $13 y$ & Larm & $\mathrm{L}$ foot $(7$ years later) & $\begin{array}{l}\text { FDG-PET: increase in BG }(R>L) / \text { motor cortex/ post } \\
\text { paracentral region; decrease in thalamus }\end{array}$ & Tremor \\
\hline $23 / M / 10$ & $\begin{array}{l}\text { Profuse bleeding at delivery, age 1:R focal seizure and R spastic } \\
\text { hemiparesis }\end{array}$ & $10 y$ & R arm/leg & None & $\begin{array}{l}\text { CT: focal atrophy adjacent to frontal horns }(L>R) \\
c / w \text { cystic encephalomalacia }\end{array}$ & R HP, dysarthria, learning disorder \\
\hline $24 / F / 17$ & Prolonged labour, delayed motor milestones & $17 y$ & $\mathrm{R}$ hand/face & None & L cerebral atrophy & R HP (face/arm), dysarthria \\
\hline $25 / M / 7$ & $\begin{array}{l}\text { Meconium at delivery, global delay, autism, ADHD, metabolic w/u } \\
\text { negative, DYT-I negative }\end{array}$ & $7 y$ & L hand/foot & L leg/arm & MRI normal & None \\
\hline $26 / F / 8$ & Jaundice $(\mathrm{R} h+)$, normal development, normal ceruloplasmin, slit lamp & $8 y$ & $L$ foot & L hand (10 years later) & $C T: R$ paraventricular lucency & None \\
\hline $27 / M / 15$ & Premature at 28 weeks, identical twin had IVH, normal development & $15 y$ & $R$ hand/foot & $\mathrm{R}$ arm $/ \operatorname{leg}$ (3 mos. later) & L basal ganglia & R HP, spasticity \\
\hline $28 / M / 6$ & TB meningitis (age 5), recovery in 4 months & 9 months & R arm & $R$ leg (9 mos. later) & I basal ganglia, porencephalic cyst & $R H P, A D H D, M R$ \\
\hline $29 / F / 2$ & Encephalitis (age 2), residual R HP, resolved & ? months & $\mathrm{R}$ hand/foot & $R$ thumb (22 years later) & No imaging & $\mathrm{R} \mathrm{HP}$, hemisensory loss \\
\hline $30 / F / 5$ & $\begin{array}{l}\text { Varicella (age 5), metabolic evaluation negative EBV, strep titres } \\
\text { negative brain biopsy c/w with Rasmussen's encephalitis CSF PCR } \\
\text { positive for rubella }\end{array}$ & 1 month & Lleg & L arm (2 months later) & $\begin{array}{l}\text { R caudate/putamen/cortical atrophy, FDG } \\
\text { PET: Marked reduction in R lateral } \\
\text { frontal/parietal/BG/thalamus }\end{array}$ & partial seizures, behavioural changes \\
\hline $31 / F / 5$ mos. & $\begin{array}{l}\text { Normal pregnancy, birth trauma motor delay, used a brace, } R \text { focal } \\
\text { seizure at } 18 \text { months, EEG: } L \text { temporal focus }\end{array}$ & 5 months & $R$ hand & R arm/leg (18 mos. later) & $\begin{array}{l}\text { Hemiatrophy of } L \text { hemisphere/hemicranium } \\
\text { porencephalic cyst (Dyke-Davidoff Mason } \\
\text { syndrome) }\end{array}$ & R HP, seizures \\
\hline $32 / F / 16$ & $\begin{array}{l}\text { Age 16: episodes of lightheadedness, headaches, L hemisensory loss, } \\
\text { HP }\end{array}$ & Unknown & L hand & L foot/arm/trunk (12 years later) & $\mathrm{R}$ thalamic AVM & L HP, hemisensory loss \\
\hline $33 / F / 8$ & $\begin{array}{l}\text { Age 8: abnormal movements of } R \text { arm/leg, Dx: thalamic tumour, } R x \\
\text { with cobalt XRT }\end{array}$ & None & R arm/leg & Face & L thalamus & Spasms \\
\hline
\end{tabular}


Table 2 Summary of clinical characteristics $(n=33)$

\begin{tabular}{|c|c|c|c|c|}
\hline & Age of onset (mean) & Latency (mean) & Site of onset (\% of cases) & Lesion site (\% of cases) \\
\hline $\begin{array}{l}\text { Poststroke } \\
(n=10)\end{array}$ & $2-69$ y $(40.5)$ & 3 months -3 y ( 9.5 months) & $\begin{array}{l}\text { Arms: } 5 / 10(50) \\
\text { Legs: } 2 / 10(20) \\
\text { Both: } 3 / 10(30)\end{array}$ & $\begin{array}{l}5 \text { basal ganglia (55) } \\
3 \text { thalamus (33) } \\
3 \text { cortical (33) }\end{array}$ \\
\hline $\begin{array}{l}\text { Post-trauma } \\
(n=8)\end{array}$ & $5-39$ y $(16.4$ y) & 2 weeks-10 y (27.5 months) & $\begin{array}{l}\text { Arms: } 5 / 8(62.5) \\
\text { Both: } 3 / 8(37.5)\end{array}$ & $\begin{array}{l}2 \text { basal ganglia }(50) \\
1 \text { cortical }(25) \\
1 \text { subcortical } \\
1 \text { midbrain }(25)\end{array}$ \\
\hline $\begin{array}{l}\text { Perinatal } \\
(n=9)\end{array}$ & $1-17$ y $(9.7$ y) & $1-17$ y $(9.7$ y) & $\begin{array}{l}\text { Arms: } 3 / 9(33) \\
\text { Legs: } 3 / 9(33) \\
\text { Both: } 3 / 9(11)\end{array}$ & $\begin{array}{l}3 \text { basal ganglia (42) } \\
1 \text { thalamus (14.3) } \\
2 \text { cortical }(28) \\
1 \text { cerebellum/pons (14.3) }\end{array}$ \\
\hline $\begin{array}{l}\text { Miscellaneous } \\
(n=6)\end{array}$ & 5 months- 16 y $(6.3$ y) & 1-9 months (4.5 months) & $\begin{array}{l}\text { Arms: } 3 / 6(50) \\
\text { Legs: } 1 / 6(16.6) \\
\text { Both: } 2 / 6(33)\end{array}$ & $\begin{array}{l}2 \text { basal ganglia }(40) \\
3 \text { thalamus }(60) \\
2 \text { cortical }(40)\end{array}$ \\
\hline
\end{tabular}

unaffected limb ranged from 2 months to 23 years (mean 6 years). Severity of dystonia was greater in the arm than the leg in $54.5 \%$ of patients, and only $9 \%$ had greater leg than arm involvement. Nineteen patients $(57 \%)$ had an associated hemiparesis which resolved or significantly improved before the onset of dystonia. Eighteen per cent of patients also had preceding hemisensory loss.

Results of neuroimaging were available in 26 patients, and one or more lesions could be identified in 25 cases. Twelve lesions were located in the contralateral basal ganglia (48\%), most commonly in the putamen. Thalamic (28\%) and cortical $(32 \%)$ lesions were also seen, and one lesion was in the contralateral midbrain. The thalamic lesions were most commonly haemorrhages and not specifically delineated as being anterior or posterior in location. Cortical lesions were also non-specific and were located in frontal, parietaloccipital, or temporal areas. One patient had basal ganglia abnormalities identified by FDG-PET scan, despite having a normal brain MRI (case 22, table 1).

On follow up telephone interview of 16 patients, most $(62.5 \%)$ thought that their symptoms were either unchanged or improved. However, six thought that they were worse, and one patient's dystonia became generalised 9 years after symptom onset. Eleven patients had either mild (seven patients) or mild-moderate (four patients) disability. Many were able to remain independent by using their non-affected side for daily activities. All patients remained ambulatory, and most (75\%) did not require assistance to ambulate.

\section{Response to treatment}

There were 158 medication trials in the 33 patients, most using a single drug ( 134 trials) and 24 trials using two or more drugs in combination. Five patients did not receive any medical therapy. Effectiveness of therapy was determined by verbal reports from patients or family members. About two thirds $(19 / 28)$ of our patients had some beneficial response to medication. However, most (95\%) required several medication trials (mean 4.7), with 54\% requiring five or more trials. Combination therapy was more effective than single drug therapy, with a beneficial response in $41.6 \%$ (10/24) compared with $23 \%$ (31/134) using monotherapy.

Overall, our patients experienced a beneficial response in only $26 \%(41 / 158)$ of medication trials. The degree of improvement as assessed by the patient was variable; 19 trials resulted in mild to moderate benefit and 22 had more marked improvement. Effectiveness was limited by side effects in $37 \%$ (15/41) of these beneficial responses, prompting a change in medication. The most common side effects were sedation, dizziness, confusion, memory loss, and blurry vision, occurring most often with benzodiazepines or anticholinergic drugs. In a follow up of 16 patients, only six continued to obtain some benefit from medications.

The most commonly used medications were trihexyphenidyl, baclofen, levodopa, clonazepam, diazepam, carbamazepine, ethopropazine, and valproic acid. The best treatment results were seen with the benzodiazepines clonazepam and diazepam, with a response rate (some improvement) of $50 \%$. Anticholinergic drugs were effective in 30\% of trials, and antiepileptic medications were only effective in $23 \%$. Both levodopa and baclofen were poorly effective, with response rates of only $12 \%$ and $6.6 \%$ respectively. Eight patients received botulinum toxin injections and half reported benefit, but in two patients this did not persist with further injections. Five of six patients who underwent thalamotomy reported benefit. However, improvement was transient in three patients, requiring reoperation, which resulted in further benefit. The duration of transient benefit was as brief as 2 months, and as long as 24 months and 5 years.

\section{Review of the literature}

We identified 158 reported cases of symptomatic hemidystonia in the literature (table 3$)$. Most were after stroke (48.5\%) or trauma $(17 \%)$, and the remainder were secondary to perinatal injury $(8.8 \%)$, tumour $(5 \%)$, infection $(5.7 \%)$, arteriovenous malfunction (4\%), and other miscellaneous causes. The mean age of onset was 25.7 years (range 3-74), and the mean latency between cerebral injury and onset of dystonia was 2.8 years (range $0-40$ ). As in our series, the longest latencies occurred in patients after perinatal injury. ${ }^{15424344}$ Sixty nine per cent were associated with hemiparesis, which usually resolved or improved as the hemidystonia developed.

Eighty seven per cent of available neuroimaging studies identified a lesion; $60 \%$ were in the basal ganglia, with the putamen and caudate most commonly affected. Lesions were also present in the thalamus (16\%), internal capsule (17.5\%), and cortex $(25 \%)$, often in association with lesions in the basal ganglia.

Data on response to treatment were available in 107 cases, and only $35 \%$ of medication trials resulted in any benefit. The anticholinergic drugs were most effective, with a beneficial response seen in $41 \%$ of treatment trials. Baclofen, benzodiazepines, and anticonvulsant drugs were less effective, with response rates of $28-30 \%$. Levodopa, dopamine agonists, and dopamine antagonists were only effective in $20 \%$ of treatment trials. Surgical intervention was beneficial in 22 of 23 (96\%) cases, but in nine of these benefit was transient. Thalamotomy was the most common procedure performed; others included 
Table 3 Review of hemidystonia literature: 158 cases from 59 references

\begin{tabular}{|c|c|c|c|c|c|}
\hline $\begin{array}{l}\text { Ref } \\
\text { (1st author) }\end{array}$ & $\begin{array}{l}\text { No of patients } \\
\text { (age in y) }\end{array}$ & Aetiology & Latency & Lesion location & $\begin{array}{l}\text { Treatment (No of } \\
\text { patients/response) (dose) }\end{array}$ \\
\hline Pettigrew ${ }^{1}$ & $\begin{array}{l}22(2-72) \\
\text { (mean 36) }\end{array}$ & $\begin{array}{l}8 \text { stroke } \\
4 \text { perinatal } \\
3 \text { trauma } \\
2 \text { storage d/o } \\
2 \text { neurodegen. } \\
2 \text { post-thalamotomy } \\
1 \text { encephalitis }\end{array}$ & 1 month-32 y & $\begin{array}{l}5 \text { basal ganglia } \\
1 \text { frontoparietal } \\
1 \text { porencephalic cyst } \\
1 \text { cerebral atrophy } \\
1 \text { putamen } \\
2 \text { internal capsule } \\
1 \text { striatum } \\
1 \text { thalamus } \\
1 \text { thalamus/basal ganglia } \\
1 \text { pallidal } \\
7 \text { normal scans }\end{array}$ & $\begin{array}{l}\text { Multiple meds (5-) } \\
\text { Levodopa (2+, 4-) } \\
\text { Trihexyphenidyl }(4+, 4-) \\
\text { Haloperidol }(2-) \\
\text { Ethopropazine (1-) } \\
\text { Propranolol (1-) } \\
\text { Levodopa/baclofen (1+) } \\
\text { Levodopa/trihexyphenidyl }(1+) \\
\text { Baclofen/trihexyphenidyl }(1+) \\
\text { Benzodiazepines (1-) } \\
\text { Amantadine (1-) } \\
\text { Thalamotomy (3tr+) }\end{array}$ \\
\hline Giroud $^{2}$ & $\begin{array}{l}18 \\
(\text { mean 10) }\end{array}$ & $\begin{array}{l}\text { Stroke } \\
14 \text { ischaemic } \\
4 \text { haemorrhagic }\end{array}$ & $?$ & 14 basal ganglia & $\begin{array}{l}\text { Ébenzodiazepines (-) } \\
\text { Baclofen (-) }\end{array}$ \\
\hline $\mathrm{Nardocci}^{3}$ & $\begin{array}{l}11 \\
\text { (mean 18.5) }\end{array}$ & $\begin{array}{l}9 \text { stroke } \\
1 \text { perinatal } \\
1 \text { antiphospholipid } \\
\text { syndrome }\end{array}$ & 1 month-9 y & $\begin{array}{l}6 \text { lenticular } \\
4 \text { IC } \\
3 \text { putamen } \\
4 \text { caudate }\end{array}$ & $\begin{array}{l}\text { Trihexyphenidyl }(1+, 4-) \\
5 \text { thalamotomies }(2+, 1+/-, \& \\
1 \mathrm{tr}+)\end{array}$ \\
\hline Dooling $^{4}$ & $4(12,20,5,2)$ & Infection & $\begin{array}{l}\text { Several } \\
\text { months-10y }\end{array}$ & $\begin{array}{l}3 \text { caudate } \\
4 \text { putamen } \\
3 \mathrm{IC} / \text { insula/temporal/ } \\
2 \mathrm{inf} \text { frontal/thalamus }\end{array}$ & Not discussed \\
\hline Burke $^{5}$ & $\begin{array}{l}5\left(7,9,6,8^{*}, 55\right) \\
{ }^{*}(\# 15 \text { in our series) }\end{array}$ & $\begin{array}{l}3 \text { perinatal } \\
1 \text { trauma } \\
1 \text { stroke }\end{array}$ & 13 months-9 y & $\begin{array}{l}1 \text { hemiatrophy } \\
1 \text { focal EEG/spikes } \\
4 \mathrm{CTs} \text { normal }\end{array}$ & $\begin{array}{l}\text { Levodopa }(\mathrm{tr}+) \\
\text { Benzhexol }(+) \\
\text { Benzhexol }(+) \\
\text { Clonazepam }(\mathrm{w}) \\
\text { Diazepam? } \\
\text { Perphenazine? }\end{array}$ \\
\hline Marsden ${ }^{9}$ & $\begin{array}{l}18(1-60) \\
\text { (mean 18) } \\
\text { *(Three cases already } \\
\text { Obeso, and Brett:see }\end{array}$ & $\begin{array}{l}2 \text { tumour } \\
2 \text { AVM } \\
6 \text { strokes } \\
3 \text { trauma } \\
1 \text { infection } \\
2 \text { anoxia } \\
2 \text { infantile epilepsy } \\
\text { reported in Narbona, } \\
\text { below) }\end{array}$ & $0-14 y$ & $\begin{array}{l}3 \text { lenticular } \\
1 \text { caudate } \\
2 \text { posterior thalamus } \\
1 \text { capsulolenticular } \\
1 \text { frontocaudate } \\
1 \text { frontolenticular/thalamus/caudate } \\
1 \text { lenticular/IC/caudate } \\
4 \text { hemiatrophy }\end{array}$ & Not discussed \\
\hline $\operatorname{Lee}^{10}$ & $\begin{array}{l}6(53,55,74,71, \\
23,4)\end{array}$ & $\begin{array}{l}4 \text { strokes } \\
1 \text { haemorrhage } \\
1 \text { trauma }\end{array}$ & 0-9 months & $\begin{array}{l}\text { Thalamus } \\
\text { Subthalamic nucleus }\end{array}$ & Levodopa (1-) \\
\hline Leiguarda ${ }^{11}$ & $\begin{array}{l}5(37,38,68,30, \\
47)\end{array}$ & $\begin{array}{l}\text { Stroke/Rx with } \\
\text { bromocriptine }\end{array}$ & Few weeks & $\begin{array}{l}\text { Frontotemporal (3) } \\
\text { Parietal (4)/lenticular (3)/ } \\
\text { Internal capsule (3) } \\
\text { Thalamus (1) }\end{array}$ & $\mathrm{d} / \mathrm{c}$ bromocriptine $(+)$ \\
\hline Kostic $^{12}$ & $4(35,47,59,26)$ & $\begin{array}{l}3 \text { strokes } \\
1 \text { AVM }\end{array}$ & $\begin{array}{l}1-2 y \\
1 y\end{array}$ & $\begin{array}{l}\text { Lenticular/IC } \\
\text { Putamen } \\
\text { Lenticular/IC/caudate } \\
\text { Temporal/caudate }\end{array}$ & Not discussed \\
\hline Rumbach $^{13}$ & $1(3)$ & Stroke & 2 months & $\begin{array}{l}\text { Cerebellum } \\
\text { Ipsilateral basal ganglia } \\
\text { SPECT: frontoparietal }\end{array}$ & $\begin{array}{l}\text { Muscle relaxants (-) } \\
\text { Anticholinergic (-) } \\
\text { Levodopa (-) } \\
\text { Thalamic DBS (-) } \\
\text { Botox (tr+) }\end{array}$ \\
\hline Ghika-Schmid $^{14}$ & ${ }^{4} 2$ (?) & Stroke & $?$ & $\begin{array}{l}\text { Thalamus/IC/lenticular } \\
\text { Parietotemporal }\end{array}$ & Not discussed \\
\hline Glatt $^{15}$ & $2(62,68)$ & $\begin{array}{l}1 \text { tumour } \\
1 \text { stroke }\end{array}$ & $\begin{array}{l}3 y \\
4 \text { months }\end{array}$ & $\begin{array}{l}\text { Parasagittal } \\
\text { Occipitotemporal/post lateral } \\
\text { thalamus }\end{array}$ & $\begin{array}{l}\text { Anticholingeric }(w) \\
\text { Dopaminergic }(w) \\
\text { Dopamine blocking agent (slight }+ \text { ) }\end{array}$ \\
\hline Lehericy $^{16}$ & $1(51)$ & Stroke & 3 weeks & $\begin{array}{l}\text { Putamen/caudate/lateral } \\
\text { pallidum/insula/IC }\end{array}$ & Not discussed \\
\hline
\end{tabular}




\begin{tabular}{|c|c|c|c|c|c|}
\hline $\begin{array}{l}\text { Ref } \\
\text { (1st author) }\end{array}$ & $\begin{array}{l}\text { No of patients } \\
\text { (age in y) }\end{array}$ & Aetiology & Latency & Lesion location & $\begin{array}{l}\text { Treatment (No of } \\
\text { patients/response) (dose) }\end{array}$ \\
\hline Burton $^{17}$ & $1(47)$ & Stroke & 9 months & Putamen/IC/caudate & Not discussed \\
\hline Hankey $^{18}$ & $1(3)$ & $\begin{array}{l}\text { Stroke-mild } \\
\text { (Moya-Moya) }\end{array}$ & $20 y$ & Lentiform nucleus (old infarct) & Levodopa (+) \\
\hline Oppenheimer' & $91(8)$ & Stroke & Hours & Putamen/caudate & Not discussed \\
\hline Demierre 20 & $3(3,7,17)$ & Stroke & $3-4$ weeks & Putamen/caudate/IC & $\begin{array}{l}\text { Anticholinergic (w) } \\
\text { Baclofen/tetrazepam (-) } \\
\text { Piribedil/baclofen/clonazepam (+) } \\
\text { Piribedil/clonazepam/dantrolene (+) } \\
\text { Benzodiazepine or dantroline (+) } \\
\text { Piribedil (+) }\end{array}$ \\
\hline Grimes $^{21}$ & $1(9)$ & Stroke & 6 months & Putamen/caudate & Not treated \\
\hline Sunohara 22 & $1(61)$ & Stroke & 3 months & $\begin{array}{l}\text { IL putamen/thalamus } \\
\mathrm{CL} \text { thalamus }\end{array}$ & $\begin{array}{l}\text { Levodopa (-) } \\
\text { Phenytoin (-) } \\
\text { Haloperidol (-) } \\
\text { Carbamazepine }(-) \\
\text { Diazepam }(\mathrm{sl} .+) \\
\text { Clonazepam }(+)(8 \mathrm{mg}) \\
\text { 5-HTP }(+)(300 \mathrm{mg})\end{array}$ \\
\hline Fross $^{23}$ & $1(47)$ & Stroke & 9 months & Putamen (MRI/PET) & not discussed \\
\hline Factor $^{24}$ & $1(18)$ & Stroke & $16 y$ & $\begin{array}{l}\text { Frontoparietal } \\
\text { Subcortical WM } \\
\text { Lentiform nucleus } \\
\text { Thalamus }\end{array}$ & Trihexyphenidyl (+) (9 mg) \\
\hline${ }^{*}$ Obeso ${ }^{25}$ & $1(22)$ & Hemiplegic migraine & 4 months & Thalamus/IC/striatum & $\begin{array}{l}\text { Pimozide (UE-, LE+) } \\
\text { Propranolol (30\%+UE) } \\
\text { Propranolol/valproic acid (+) } \\
\text { Propranolol/clonazepam (+) }\end{array}$ \\
\hline Giroud $^{26}$ & $1(9)$ & Stroke & 21 days & Caudate/IC/putamen & Benzodiazepines/dantrolene (?) \\
\hline Krystkowiak ${ }^{27}$ & $2(39,52)$ & Stroke & $\begin{array}{l}6 \text { weeks, } \\
4 \text { months }\end{array}$ & $\begin{array}{l}\text { Putamen/caudate/Gpe } \\
\text { Caudate/putamen/pallidum/ } \\
\text { thalamus }\end{array}$ & Not discussed \\
\hline Fernande $z^{28}$ & $1(38)$ & Stroke & 18 weeks & Right midbrain & $\begin{array}{l}\text { Baclofen }(-)(30 \mathrm{mg}) \\
\text { Benztropine (-) }(0.5 \mathrm{mg}) \\
\text { Amantadine (-) } \\
\text { Valproic acid (-) } \\
\text { Levodopa (-) } \\
\text { Pimozide (-) } \\
\text { Perphenazine (-) } \\
\text { Mexilitine (-) } \\
\text { Carbamazepine (-) } \\
\text { Phenobarbital (-) } \\
\text { Clozapine (-) } \\
\text { Pallidal DBS (transient +) }\end{array}$ \\
\hline Takahashi $^{29}$ & $1(5)$ & Stroke & None & Caudate/putamen & $\begin{array}{l}\text { Haloperidol (-) }(1 \mathrm{mg}) \\
\text { Levodopa (-) } \\
\text { Diazepam (-) } \\
\text { Dantrolene (-) }\end{array}$ \\
\hline Gille $^{30}$ & $1(68)$ & Stroke & 8 months & Posterolateral thalamus & Not discussed \\
\hline Krauss $^{31}$ & $1(9)$ & Stroke/trauma & Months & $\begin{array}{l}\text { Parieto-occipital lobe } \\
\text { Insula/putamen }\end{array}$ & Thalamotomy (transient +) \\
\hline $\operatorname{Lee}^{32}$ & $5(6,12,24,21,21)$ & Trauma & $\begin{array}{l}\text { Several } \\
\text { months-9 y } \\
\text { (mean } 2 \text { y) }\end{array}$ & $\begin{array}{l}\text { Lenticular/IC/thalamus } \\
\text { Posterolateral thalamus } \\
\text { Lenticular/IC } \\
\text { Inferior frontal } \\
\text { None in one case }\end{array}$ & $\begin{array}{l}\text { Clonazepam (+) } \\
\text { Trihexyphenidyl (-) } \\
\text { Levodopa (-) } \\
\text { Propranolol (-) } \\
\text { Carbamazepine (-) } \\
\text { Tetrabenazine (-) } \\
\text { Baclofen (-) }\end{array}$ \\
\hline
\end{tabular}




\begin{tabular}{|c|c|c|c|c|c|}
\hline $\begin{array}{l}\text { Ref } \\
\text { (1st author) }\end{array}$ & $\begin{array}{l}\text { No of patients } \\
\text { (age in y) }\end{array}$ & Aetiology & Latency & Lesion location & $\begin{array}{l}\text { Treatment (No of } \\
\text { patients/response) (dose) }\end{array}$ \\
\hline Krauss $^{33}$ & $\begin{array}{l}6(8,13,6,6,13 \\
18)\end{array}$ & Trauma & $\begin{array}{l}3 \text { months }-4 \mathrm{y} \\
\text { (Mean } 1.8 \mathrm{y} \text { ) }\end{array}$ & $\begin{array}{l}3 \text { caudate } \\
6 \text { putamen } \\
3 \text { pallidum } \\
\mathrm{CL} \text { hemiatrophy }\end{array}$ & $\begin{array}{l}\text { Trihexyphenidyl, pimozide, } \\
\text { tetrabenazine, methlydopa (-) } \\
\text { Dantrolene, diazepam, bromazepam } \\
\text { (+) } \\
\text { Trihexyphenidyl (+) } \\
\text { Chlormezepam, phenytoin, biperidin, } \\
\text { haldol (-) } \\
\text { Thalamotomy (most transient + but } \\
\text { three sustained +) }\end{array}$ \\
\hline Eaton $^{34}$ & $1(56)$ & Trauma & 10 weeks & L.subdural haematoma & Removal of haematoma (+) \\
\hline Mauro $^{35}$ & $2(7,8)$ & Trauma & $\begin{array}{l}2 \text { weeks-few } \\
\text { days }\end{array}$ & 1 caudate, 1 putamen & Carbamazepine/trihexyphenidyl (-) \\
\hline Andrew ${ }^{36}$ & $1(8)$ & Trauma & 1 day & Caudate/IC/lenticular nucleus & Thalamotomy (+) \\
\hline${ }^{*} \mathrm{Brett}^{37}$ & $1(5)$ & Trauma & 1 week & Basal ganglia & Not discussed \\
\hline Perlmutter ${ }^{38}$ & $1(50)$ & Trauma & $20 \mathrm{~min}$ & $\begin{array}{l}\text { Basal ganglia by PET } \\
\text { CT normal }\end{array}$ & $\begin{array}{l}\text { Clonazepam/iv benztropine (-) } \\
\text { Levodopa (w) } \\
\text { Haloperidol (tr+) } \\
\text { Phenytoin (+) } \\
\text { Trihexyphenidyl (+) } \\
\text { Phenytoin/trihexyphenidyl (++) }\end{array}$ \\
\hline $\mathrm{O}^{\prime}$ Callaghan ${ }^{39}$ & $1(12)$ & Trauma & $\sim 2 y$ & $\mathrm{~N} / \mathrm{A}$ & $\begin{array}{l}\text { Pallidotomy }(\mathrm{tr}+) \\
\text { Thalamotomy }(+)\end{array}$ \\
\hline Sella ${ }^{40}$ & $1(16)$ & $\begin{array}{l}\text { Trauma (extradural } \\
\text { haematoma) }\end{array}$ & 2 months & Thalamus/IC & $\begin{array}{l}\text { Baclofen }(-)(50 \mathrm{mg}) \\
\text { Levodopa }(-)(1 \mathrm{~g}) \\
\text { Haloperidol }(-)(5 \mathrm{mg}) \\
\text { Clonazepam }(-)(3 \mathrm{mg}) \\
\text { 5-HTP (-) (300 mg) } \\
\text { Thalamic DBS (+) }\end{array}$ \\
\hline Kellner $^{41}$ & $1(63)$ & Trauma & $1 y$ & $\begin{array}{l}\text { Lentiform nucleus } \\
\text { Frontoparietal WM }\end{array}$ & Not discussed \\
\hline Midgard $^{42}$ & $1(13)$ & Perinatal & $13 y$ & $\begin{array}{l}\text { Striatal atrophy } \\
\text { (putamen/caudate) }\end{array}$ & $\begin{array}{l}\text { Diazepam (-), alcohol (+), } \\
\text { Propranolol/tetrabenazine (-) } \\
\text { Trihexyphenidyl (+) }\end{array}$ \\
\hline Saint-Hilaire ${ }^{43}$ & $2(8,15)$ & Perinatal & $8-15 y$ & $\mathrm{CL}$ hemiatrophy (CT) & Anticholinergics (+) \\
\hline Treves $^{44}$ & $1(40)$ & Perinatal & $40 y$ & $\mathrm{~N} / \mathrm{A}$ & Carbamazepine (+) \\
\hline Krauss $^{45}$ & $2(9,15)$ & Astrocytoma & None & Basal ganglia or thalamus & Steroids $(-)$ \\
\hline Sciarra ${ }^{46}$ & $1(15)$ & Tumour & ? & Basal ganglia/thalamus & Surgical resection (+) \\
\hline Krauss $^{47}$ & $1(57)$ & Tumour & ? & $\begin{array}{l}\text { Parieto-occipital } \\
\text { Compression of basal ganglia }\end{array}$ & Surgical resection (+) \\
\hline${ }^{*}$ Narbona $^{48}$ & 1 (8) & Tumour, astrocytoma & $?$ & Putamen & $\begin{array}{l}\text { Haloperidol (-) } \\
\text { Benzhexol (-) } \\
\text { Chlorpromazine (-) } \\
\text { Levodopa (-) } \\
\text { Carbamazepine (+) } \\
\text { XRT (slight }+, 10 \%)\end{array}$ \\
\hline Vandertop ${ }^{49}$ & $1(8)$ & Tumour & ? & Chiasm/hypothalamus & Not discussed \\
\hline Gordin $^{50}$ & $1(22)$ & Encephalitis & Days & Putamen/pallidum & Luminal/somnifen (-) \\
\hline Micheli ${ }^{51}$ & $1(11)$ & $\begin{array}{l}\text { Infection } \\
\text { (toxoplasmosis) }\end{array}$ & 4 days & $\begin{array}{l}\text { None } \\
\text { CT/MRI normal }\end{array}$ & $\begin{array}{l}\text { Trihexyphenidyl (-) } \\
\text { Delorazepam (-) } \\
\text { Antibiotics for toxoplasmosis (+) }\end{array}$ \\
\hline Burband $^{52}$ & $1(14)$ & Herpes zoster arteritis & 6 weeks & Putamen/caudate & $\begin{array}{l}\text { Trihexyphenidyl/levodopa }(-) \\
\text { Baclofen }(+) \\
\text { Botox }(+)\end{array}$ \\
\hline
\end{tabular}




\begin{tabular}{|c|c|c|c|c|c|}
\hline $\begin{array}{l}\text { Ref } \\
\text { (1 st author) }\end{array}$ & $\begin{array}{l}\text { No of patients } \\
\text { (age in y) }\end{array}$ & Aetiology & Latency & Lesion location & $\begin{array}{l}\text { Treatment (No of } \\
\text { patients/response) (dose) }\end{array}$ \\
\hline Caviness $^{53}$ & $1(45)$ & Sarcoidosis & $3 y$ & $\begin{array}{l}\text { None } \\
\text { MRI normal }\end{array}$ & $\begin{array}{l}\text { Intravenous steroids (+) } \\
\text { Levodopa (slight }+ \text { ) } \\
\text { Pergolide } \\
\text { Prednisone } \\
\text { Baclofen } \\
\text { Levodopa/clonazepam (+) }\end{array}$ \\
\hline Awaad $^{54}$ & $1(3)$ & Glutaric aciduria & 3 years & $\begin{array}{l}\text { FDG-PET: decrease in } \\
\text { striatum/frontal cortex/insula } \\
\text { MRI: bifrontal temporal atrophy }\end{array}$ & Baclofen (+) $80 \%$ \\
\hline $\begin{array}{l}\text { Jimenez- } \\
\text { Jiminez } z^{55}\end{array}$ & $1(47)$ & Thiethylperazine & None & $\begin{array}{l}\text { None } \\
\mathrm{CT} / \mathrm{MRI} \text { normal }\end{array}$ & biperiden $(+)$ \\
\hline Angelini $i^{56}$ & $3(7,12,14)$ & $\begin{array}{l}\text { Antiphospholipid } \\
\text { syndrome (stroke) }\end{array}$ & 0-3 months & $\begin{array}{l}\text { Posterior IC } \\
\text { putamen } \\
\text { putamen/caudate }\end{array}$ & Prednisone (1-) \\
\hline Friedman $^{57}$ & $2(14,24)$ & $\mathrm{AVM}$ & ? & $\begin{array}{l}\text { basal ganglia } \\
\text { temporoparietal }\end{array}$ & Anticholinergics (1+) \\
\hline Leenders ${ }^{58}$ & $1(7)$ & Congenital (?AVM) & ? & $\begin{array}{l}\text { Brain stem/thalamus } \\
\text { F-dopa PET: CL striatum }\end{array}$ & Trihexyphenidyl (+) \\
\hline Lees $^{59}$ & $1(7)$ & ? & $1 y$ & N/A & $\begin{array}{l}\text { Smoking (tr+30 } \mathrm{min}) \\
\text { Benzhexol }(+) \\
\text { Benzodiazepines }(-) \\
\text { Nicotine }(\mathrm{tr}+1 \mathrm{~h})\end{array}$ \\
\hline Quinn ${ }^{60}$ & $1(7)$ & ? & ? & $\begin{array}{l}\text { L. basal ganglia } \\
\text { Bilateral midbrain }(L>R)\end{array}$ & Not discussed \\
\hline
\end{tabular}

+, Beneficial response; -, no response; tr+, transient response; w, worsening of dystonia; CL, contralateral, IL, ipsilateral; UE, upper extremity; LE, lower extremity; IC, internal capsule; WM, white matter; Gpe, external globus pallidum; N/A, not available; ?, unknown; 5-HTP, 5-hydroxytryptophan.

thalamic deep brain stimulation (two), pallidotomy (one), and pallidal deep brain stimulation (one). Botulinum toxin injections were beneficial in two of two cases.

\section{DISCUSSION}

This retrospective study describes the clinical features and response to treatment in hemidystonia. Our primary aim was to determine the most effective treatment strategy for patients with hemidystonia. There are several limitations to our study. Firstly, in our series many medication trials occurred before the initial visit to our centre, and response to therapy therefore relied on patients' recall. Secondly, most patients were only evaluated at a single office visit, and subsequent response to treatment was primarily determined by follow up telephone calls. We recognise that relying on patients' reports of improvements does not account for a placebo effect. However, we chose to use patients' accounts of treatment response because we were primarily interested in determining which medications patients thought helped them the most. A more objective assessment of response to treatment was precluded by the very limited number of follow up evaluations. Many patients were seen several years ago with most being lost to follow up; only two patients were able to return for another physical examination.

Despite these limitations, we were able to draw several conclusions about the nature of hemidystonia. The most common lesion resulting in hemidystonia occurs in the contralateral basal ganglia. The most common aetiology is stroke, occurring in $48.7 \%$ of the cases in the literature and in almost $30 \%$ of the patients in our series. Perinatal injury and trauma were nearly as common as stroke in our series, but were less common in the literature. Hemidystonia is rarely a consequence of basal ganglia stroke in adults, ${ }^{61}{ }^{62}$ occurring more often in younger patients. Most patients had onset of hemidystonia at less than
25 years of age, supporting the idea that the younger brain is more susceptible to developing hemidystonia.

There is often a delay between the suspected cerebral insult and the onset of hemidystonia, occurring up to 17 years after the initial injury in our series and as long as 40 years $^{44}$ in the literature. Leenders et al proposed that the delayed development of dystonia may be related to "slowly evolving" aberrant neuronal sprouting. ${ }^{58}$ In our review, the latency was longest when hemidystonia was secondary to perinatal injury. This may be related to the greater ability of the developing brain to recover from cerebral insults, or secondary to a delayed recognition of this syndrome in young children.

On follow up evaluation, dystonia had stabilised in most of our patients; none had complete resolution of dystonia. The course of hemidystonia is rarely discussed in the literature. However, Pettigrew and Jankovic reported on one patient who had spontaneous resolution of hemidystonia after 4 years, and another had resolution of dystonia after 3 months of treatment with levodopa and trihexyphenidyl. ${ }^{1}$ A handful of reports also describe progression of dystonia over months to years followed by stabilisation..$^{3235} 4258$ Thus, the natural history of hemidystonia seems to be initial progression with spread over months to years, followed by eventual stabilisation.

Based on our review, hemidystonia responds poorly to medical therapy. In our series of patients, more than one third of effective medication trials were discontinued because of side effects, and nearly $50 \%$ were only partially effective. Benzodiazepines were the most effective medications in our patients. Anticholinergic drugs were less effective, with a response rate of only $30 \%$. In the literature, anticholinergic drugs were the most effective treatment, with a response rate of $41 \%$, similar to previous reports of dystonia therapy.$^{63}$ Fahn, however, reported five cases of hemidystonia with no response to high dose anticholinergic treatment. In Fahn's series of 
patients, those with symptomatic dystonia were less responsive to treatment than idiopathic cases of dystonia with only $36 \%$ responding to treatment compared with $59 \%$ in the idiopathic group. ${ }^{6}$ This would suggest that patients with symptomatic dystonia do not respond to medical treatment as well as idiopathic cases of dystonia. However, in the report of Marsden et al, treatment response of severe dystonia to high dose anticholinergic drugs was independent of aetiologythat is, there was no difference between the response to treatment between idiopathic and symptomatic cases.$^{64}$ The difference between these two studies is difficult to interpret because in both of them there were many fewer symptomatic cases than idiopathic cases. This small sample size may not have allowed for a relevant comparison between the two treatment groups.

Surgical intervention was more successful than medical therapy, both in our series and in the literature. In our patients, results were transient in $60 \%$ prompting repeat procedures, whereas in the literature $41 \%$ had a transient result. Transient benefit from thalamotomy or pallidotomy suggests that the target is correct but the size of the lesion may be too small. Deep brain stimulation, either in the thalamus or the internal pallidum, may be more successful at producing a longer lasting result as the stimulation parameters can be adjusted to produce maximal benefit. Recently there have been several reports of success using deep brain stimulation of the internal globus pallidus for generalised dystonia, ${ }^{65-68}$ and even for post-traumatic hemidystonia. ${ }^{69}$ There have been no studies directly comparing thalamic versus pallidal deep brain stimulation for dystonia but as there has been much more experience recently with pallidal deep brain stimulation, we can only speculate that this would be the surgical treatment of choice. However, we think that a randomised study comparing the two techniques would be necessary to determine the most effective surgery for hemidystonia.

In conclusion, hemidystonia usually occurs in the setting of a structural lesion of the contralateral basal ganglia or thalamus. Stroke is the most common cause, followed by trauma and perinatal injury. Hemidystonia typically progresses over years followed in most cases by stabilisation. Response to medical therapy is generally poor and often limited by side effects. However, many patients may benefit from benzodiazepines or anticholinergic drugs. Surgical intervention may be more successful, but results can be transient. Thalamic or pallidal deep brain stimulation may be a reasonable alternative to lesioning. Prospective double blind placebo controlled studies are needed to better evaluate both medical and surgical treatments for hemidystonia.

\section{Authors' affiliations}

C Chuang, S Fahn, S J Frucht, The Neurological Institute, Columbia-Presbyterian Medical Center, 710 West 168th Street, New York, NY 10032, USA

\section{REFERENCES}

1 Pettigrew L, Jankovic J. Hemidystonia: a report of 22 patients and a review of the literature. J Neurol Neurosurg Psychiatry 1985;48:650-7.

2 Giroud M, Lemesle M, Madinier G, et al. Stroke in children under 16 years of age. Clinical and etiological difference with adults. Acta Neurol Scand 1997;96:401-6.

3 Nardocci N, Zorzi G, Grisoli M, et al. Acquired hemidystonia in childhood: a clinical and neuroradiological study of 13 patients. Pediatr Neurol 1996:15:108-13.

4 Dooling EC, Adams RD. The pathological anatomy of posthemiplegic athetosis. Brain 1975;98:29-48

5 Burke RE, Fahn S, Gold AP. Delayed-onset dystonia in patients with "static" encephalopathy. J Neurol Neurosurg Psychiatry 1980;43:789-97.

6 Fahn S. High dosage anticholinergic therapy in dystonia. Neurology 1983;33:1255-61

7 Andrew J, Fowler CJ, Harrison MG. Stereotaxic thalamotomy in 55 cases of dystonia. Brain 1983;106:981-1000.

8 Burke RE, Fahn S, Marsden CD, et al. Validity and reliability of a rating scale for the primary torsion dystonias. Neurology 1985;35:73-7.
9 Marsden CD Obeso JA, Zarranz JJ, et al. The anatomical basis of symptomatic hemidystonia. Brain 1985; 108:463-83.

10 Lee MS, Marsden CD. Movement disorders following lesions of the thalamus or subthalamic region. Mov Disord 1994;9:493-507.

11 Leiguarda R, Merello M, Sabe L, et al. Bromocriptine-induced dystonia in patients with aphasia and hemiparesis. Neurology 1993;43:231922.

12 Kostic VS, Stojanovic-Svetel M, Kacar A. Symptomatic dystonias associated with structural brain lesions: report of 16 cases. Can J Neurol Sci 1996;23:53-6

13 Rumbach L, Barth P, Costaz A, et al. Hemidystonia consequent upon ipsilateral vertebral artery occlusion and cerebellar infarction. Mov Disord 1995;10:522-5.

14 Ghika-Schmid F, Ghika J, Regli F, et al. Hyperkinetic movement disorders during and after acute stroke: the Lausanne stroke registry. $J$ Neurol Sci 1997;146:109-16.

15 Glatt SL, Nausieda PA. Posthemiplegic dystonia: radiographic and pharmacologic analysis. Neurology 1984;34:290-1.

16 Lehericy S, Vidailhet M, Dormont D, et al. Striatopallidal and thalamic dystonia: a magnetic resonance imaging anatomoclinical study. Arch Neurol 1996:53:241-50

17 Burton K, Farrell K, Li D, et al. Lesions of the putamen and dystonia: CT and magnetic resonance imaging. Neurology 1984;34:962-5.

18 Hankey GJ, Sadka M, Late-onset post-hemiplegic hemidystonia: CT and angiographic findings. Aust NZJ Med 1989;19:147-50.

19 Oppenheimer DR. A case of striatal hemiplegia. J Neurol Neurosurg Psychiatry 1967;30:134-9.

20 Demierre B, Rondot P. Dystonia caused by putamino-capsulo-caudate vascular lesions. J Neurol Neurosurg Psychiatry 1983;46:404-9.

21 Grimes JD, Hassan MN, Quarrington AM, et al. Delayed-onset posthemiplegic dystonia: CT demonstration of basal ganglia pathology. Neurology 1982;32:1033-5.

22 Sunohara N, Mukoyama M, Mano Y, et al. Action-induced rhythmic dystonia: an autopsy case. Neurology 1984;34:321-7.

23 Fross RD, Martin WRW, Li D, et al. Lesions of the putamen: their relevance to dystonia. Neurology 1987:37:1125-9.

24 Factor SA, Sanchez-Ramon J, Weiner WJ. Delayed-onset dystonia associated with corticospinal tract dysfunction. Mov Disord 1988;3:201-10

25 Obeso JA, Martinez Vila E, Delgado G, et al. Delayed onset dystonia following hemiplegic migraine. Headache 1984;24:266-8.

26 Giroud M, Dumas R. Dystonia after putamino-capsular-caudate infarction in childhood. Rev Neurol 1988;5:375-7.

27 Krystkowiak P, Martinat P, Defebvre L, et al. Dystonia after striatopallidal and thalamic stroke: clinicoradiological correlations and pathophysiological mechanisms. J Neurol Neurosurg Psychiatry 1998:65:703-8

28 Fernandez HH, Friedman JH, Centofanti JV. Benedikt's syndrome with delayed-onset rubral tremor and hemidystonia: a complication of tic douloureux surgery. Mov Disord 1999;14:695-7.

29 Takahashi S, Oki J, Miyamoto A, et al. Hemidystonia, hemichorea, and motor aphasia associated with bilateral ischemic lesions in the striatum: regional cerebral blood flow studies to clarify the pathophysiology. $J$ Child Neurol 1998;13:408-11.

30 Gille M, Van den Bergh P, Ghariani S, et al. Delayed-onset hemidystonia and chorea following contralateral infarction of the posterolateral thalamus. A case report. Acta Neurol Belg 1996;96:307-11.

31 Krauss JK, Jankovic J. Hemidystonia secondary to carotid artery gunshot injury. Childs Nervous System 1997;13:285-8.

32 Lee MS, Rinne JO, Ceballos-Baumann A, et al. Dystonia after head trauma. Neurology 1994;44:1374-8.

33 Krauss JK, Mohadjer M, Braus DF, et al. Dystonia following head trauma: a report of nine patients and review of the literature. Mov Disord 1992; 7:263-72.

34 Eaton JM. Hemidystonia due to subdural hematoma. Neurology 1988;38:507.

35 Mauro AJ, Fahn S, Russman B. Hemidystonia following "minor" head trauma. Ann Neurol 1980;8:108

36 Andrew J, Fowler C, Harrison MG. Hemi-dystonia due to focal basal ganglia lesion after head injury and improved by stereotaxic thalamotomy. I Neurol Neurosurg Psychiatry 1982:45:276.

37 Brett EM, Hoare RD, Sheehy MP, et al. Progressive hemi-dystonia due to focal basal ganglia lesion after mild head trauma. J Neurol Neurosurg Psychiatry 1981;44:460

38 Perlmutter JS, Raichle ME. Pure hemidystonia with basal ganglion abnormalities on positron emission tomography. Ann Neurol 1984;15:228-33

39 O'Callaghan ED. Torsion dystonia complicating childhood hemiplegia. Med J Aust 1962;49:465-8.

40 Sellal F, Hirsch E, Barth $\mathrm{P}$, et al. A case of symptomatic hemidystonia improved by ventroposterolateral thalamic electrostimulation. Mov Disord 1993;8:515-18.

41 Kellner MB, Strian F. Bizarre delusion and post-hemiplegic hemidystonia. Br J Psychiatry 1991;159:448.

42 Midgard R, Aarli JA, Julsrud OJ, et al. Symptomatic hemidystonia of delayed onset. Magnetic resonance demonstration of pathology in the putamen and the caudate nucleus. Acta Neurol Scand 1989;79:27-31.

43 Saint-Hilaire MH, Burke RE, Bressman SB, et al. Delayed-onset dystonia due to perinatal or early childhood asphyxia. Neurology 1991;41:216-22.

44 Treves T, Korczyn AD. Progressive dystonia and paraparesis in cerebral palsy. Eur Neurol 1986;25: 148-53. 
45 Krauss JK, Braus DF, Mohadjer M, et al. Evaluation of the effect of treatment on movement disorders in astrocytomas of the basal ganglia and the thalamus. J Neurol Neurosurg Psychiatry 1993;56:11 13-18.

46 Sciarra D, Sprofkin BE. Symptoms and signs referable to the basal ganglia in brain tumor. Arch Neurol Psychiatry 1953;69:450-61

47 Krauss JK, Mohadjer M, Nobbe F, et al. Hemidystonia due to a contralateral parieto-occipital metastasis: disappearance after removal of the mass lesion. Neurology 1991;41:1519-20.

48 Narbona J, Obeso JA, Tunon T, et al. Hemi-dystonia secondary to localised basal ganglia tumour. J Neurol Neurosurg Psychiatry 1984:47:704-9.

49 Vandertop WP, Kamphuis DJ, Witkamp TD. Hemidystonia as presenting symptom of an optic glioma. Childs Nerv Syst 1997;13:289-92.

50 Gordin R. A case of unilateral torsion-dystonia. J Nerv Ment Dis 1939:90:344-57.

51 Micheli R, Perini A, Duse M. Hemidystonia secondary to acquired toxoplasmosis in a non-immunodeficient patient. Eur J Pediatr 1994; 153:731-3.

52 Burband R, Berge J, Lagueny A, et al. Delayed-onset hemidystonia secondary to herpes zoster ophthalmicus-related intracerebral arteritis in an adolescent. J Neurol 1997;244:470-2.

53 Caviness JN, Knox CA. Hemidystonia occurring in a patient with sarcoidosis. Mov Disord 1996; 1 1:340-1.

54 Awaad Y, Shamato H, Chugani H. Hemidystonia improved by baclofen and PET scan findings in a patient with glutaric aciduria type I. J Child Neurol 1996;11:167-9.

55 Jimenez-Jimenez FJ, Vazquez A, Garcia-Ruiz P, et al. Chronic hemidystonia following acute dystonic reaction to thiethylperazine. $J$ Neurol Neurosurg Psychiatry 1991;54:562.

56 Angelini L, Rumi V, Naradocci N, et al. Hemidystonia symptomatic of primary antiphospholipid syndrome in childhood. Mov Disord 1993;8:383-6

57 Friedman DI, Jankovic J, Rolak LA. Arteriovenous malformation presenting as hemidystonia. Neurology 1986;36:1590-3.
58 Leenders KL, Frackowiak RSJ, Quinn N, et al. Ipsilateral blepharospasm and contralateral hemidystonia and parkinsonism in a patient with a unilateral rostral brainstem-thalamic lesion: structural and functional abnormalities studied with CT, MRI, and PET scanning. Mov Disord 1986;1:51-8.

59 Lees AJ. Hemidystonia relieved by nicotine. Lancet 1984;ii:871.

60 Quinn N, Bydder G, Leenders N, et al. Magnetic resonance imaging to detect deep basal gangli lesions in hemidystonia that are missed by computerised tomography. Lancet 1985;ii:1007-8.

61 Damasio AR, Damasio H, Rizzo M, et al. Aphasia with non-hemorrhagic lesions in basal ganglia and internal capsule. Arch Neurol 1982;39:15-20.

62 Naeser MA Alexander MP, Helm-Estabrooks N, et al. Aphasia with predominantly subcortical lesion sites: description of three capsular/putaminal aphasia syndromes. Arch Neurol 1982;39:2-14.

63 Fahn S. Systemic therapy of dystonia. Can J Neurol Sci 1987; 14:528.

64 Marsden CD, Marion M-H, Quinn N. The treatment of severe dystonia in children and adults. J Neurol Neurosurg Psychiatry 1984;47:116673.

65 Courbes P, Echenne B, Roubertie A, et al. Traitement de la dystonie generalisee a debut precoce par stimulation chronique bilaterale des globus pallidus internes. Neurochirurgie 1999;45:139-44

66 Courbes P, Roubertie A, Vayssiere N, et al. Treatment of DYT-1-generalised dystonia by stimulation of internal globus pallidus. Lancet 2000;355:2220-1.

67 Kumar R, Dagher A, Hutchison WD, et al. Globus pallidus deep brain stimulation for generalized dystonia: clinical and PET investigation. Neurology 1999;53:871-4

68 Tronnier VM, Fogel W. Pallidal stimulation for generalized dystonia. Report of three cases. J Neurosurg 2000;92:453-6.

69 Loher TJ, Hasdemir MG, Burgunder J-M, et al. Long-term follow-up study of chronic globus pallidus internus stimulation for posttraumatic hemidystonia. J Neurosurg 2000;92:457-60.

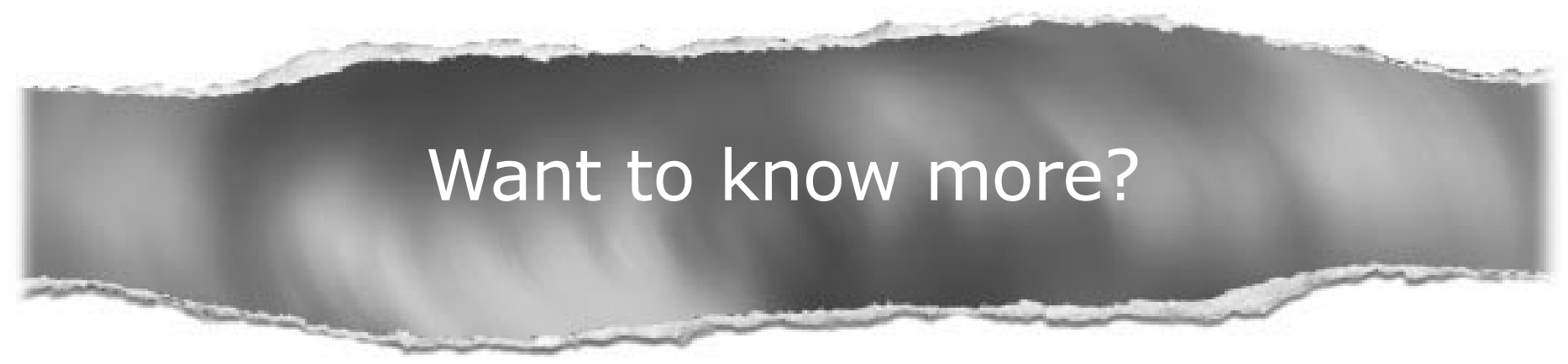

\section{Data supplements}

Limited space in printed journals means that interesting data and other material are often edited out of articles; however, limitless cyberspace means that we can include this information online.

Look out for additional tables, references, illustrations.

www.jnnp.com 